\section{Exploiting Directional Antennas for Reduced-Dimension Space-Time RAKE Receiving}

Peng Jin, Nathan A. Goodman, and Kathleen L. Melde

\begin{abstract}
We propose to combine directional antennas in a conformal array configuration with a matching two-stage receiver architecture to improve performance of code-division multiple-access (CDMA) communication systems in time-varying channels. We propose a reduced-dimension space-time (ST) RAKE architecture that matches the ST correlation structure of the data received by a circular array. In time-varying channels, this reduced-dimension architecture outperforms the full-dimension architecture due to improved convergence of its adaptive processor.
\end{abstract}

Index Terms-Multipath, multiuser detection, RAKE receiver, spacetime beamforming.

\section{INTRODUCTION}

Multipath fading and multiple access interference (MAI) are two major factors that affect the performance of code-division multipleaccess (CDMA) [1] communication systems. MAI causes the near-far effect [2], where interfering users are received with much higher power than the desired user, which leads to a poor signal-to-interference ratio even after the despreading process. Multipath fading can cause intersymbol interference, but the frequency selectivity of multipath environments can be exploited by the RAKE receiver [1], [3] by combining individual multipath components in time. The multiuser RAKE receiver [4], [5] rejects MAI while exploiting multipath diversity.

Although the multiuser RAKE receiver is designed to reduce MAI, finite-length spreading codes cannot be mutually orthogonal over all arbitrary time delays. Thus, even the receivers in [4] and [5] can suffer from the near-far effect. Fortunately, additional resistance to the near-far effect can be obtained by employing antenna arrays. Some papers demonstrating the benefits of antenna arrays in CDMA are [6]-[8]. Other space-time (ST) receivers with improved performance have been presented in [9]-[15] using antenna arrays with symbol- or chip-level equalization. While the said ST receivers greatly alleviate the near-far effect, they have much higher dimensionality than a single-antenna system, which creates computational and convergence issues that degrade the signal-to-interference-plus-noise ratio (SINR) when the interference statistics must be estimated from training data. Reduced-rank array processing has been proposed in [16] and [17] to ease this dimensionality problem.

In this paper, we propose a reduced-dimension architecture that matches and exploits the natural correlation structure of conformal antenna arrays employing directive antennas. While the aforementioned papers present excellent results in multiuser wireless communication, they give little attention to the effects of antenna directivity and antenna array structure. In this paper, the effects of directive antennas and array structure on ST RAKE receivers in direct-sequence CDMA (DS-CDMA) communication are studied. In particular, we seek to exploit the localized spatial correlation structure created by the pattern diversity [18] of circular arrays with individually directive antennas.

Manuscript received July 5, 2006; revised July 6, 2007, October 5, 2007 , November 6, 2007, and November 7, 2007. First published February 15, 2008; current version published November 12, 2008. This work was supported in part by the National Science Foundation under Grant ECS-0098547. The review of this paper was coordinated by Dr. C. Ling.

The authors are with the Department of Electrical and Computer Engineering, University of Arizona, Tucson, AZ 85721-0104 USA.

Digital Object Identifier 10.1109/TVT.2008.918710
To exploit this special correlation structure, we propose a matching two-stage reduced-dimension architecture. The first stage consists of multiple reduced-dimension receivers in parallel. In the second stage, the outputs of the reduced-dimension receivers are combined to maximize the output SINR. Since the amount of data required for training an adaptive receiver is proportional to the dimension of the receiver [19], fewer training data are needed for the reduced-dimension receiver, resulting in improved convergence.

This paper is organized as follows: Section II presents the data model for the ST receiver and describes ST beamforming (BF). In Section III, performance comparisons between linear and circular arrays for both omnidirectional and directional antennas are presented. The reduced-dimension ST receiver and simulation results for timeinvariant and time-varying channels are presented in Section IV. Section V concludes this paper.

\section{SIGNAL MODEL}

Let $\mathbf{r}(t)$ be an $M$-dimensional vector representing the baseband signal received by the $M$ antennas of a receiving array. Using a notation similar to that of [9], $\mathbf{r}(t)$ can be represented as

$$
\begin{aligned}
\mathbf{r}(t)=\sum_{k=1}^{K} \sum_{l=1}^{L_{k}} \sqrt{P_{k}} \beta_{k}^{l} \mathbf{g}\left(\theta_{k}^{l}\right) \\
\quad \times \sum_{i=-\infty}^{\infty}\left\{b_{k}(i) c_{k}\left(t-i T_{b}-\tau_{k}^{l}\right)\right\}+\mathbf{n}(t)
\end{aligned}
$$

where $K$ is the number of users, $L_{k}$ is the number of multipath components arriving at the receiver for the $k$ th user, $b_{k}(n)$ is the $n$th symbol value for the $k$ th user, and $c_{k}(t)$ is the spreading waveform for the $k$ th user. In addition, in (1), $P_{k}$ is the power of user $k$, and $\beta_{k}^{l}, \theta_{k}^{l}$, and $\tau_{k}^{l}$ are the complex path gain, angle of arrival (AOA), and delay, respectively, for the $l$ th path of the $k$ th user. It is assumed that each user is assigned a code of $N_{c}$ chips of duration $T_{c}$ such that the duration of a symbol is $T_{b}=N_{c} T_{c}$. Finally, $\mathbf{n}(t)$ is an $M$-dimensional vector of circularly symmetric complex white (both in time and space) Gaussian noise with zero mean and power spectral density $N_{0} / 2$. In this paper

$$
\mathbf{g}(\theta)=\mathbf{a}(\theta) \circ \mathbf{f}(\theta)
$$

where $\mathbf{f}(\theta)$ is the multichannel antenna element pattern [20], $\mathbf{a}(\theta)$ is the array response vector [20] representing the relative phase shift at each receiving antenna due to a plane wave arriving from angle $\theta$, and "o" is the elementwise product known as the Hadamard product. For a plane wave with wavenumber $\mathbf{k}(\theta)=k_{x}(\theta) \hat{x}+k_{y}(\theta) \hat{y}$, the $m$ th element of the array response vector is $a_{m}=e^{-j \mathbf{k}(\theta) \cdot \mathbf{r}_{m}}$, where $\mathbf{r}_{m}$ is the location of the $m$ th antenna's phase center. In the aforementioned previous work, $\mathbf{g}(\theta)=\mathbf{a}(\theta)$, and there are no discussions of the effects of directive antenna elements. In our work, by incorporating the antenna pattern $\mathbf{f}(\theta)$, we are able to exploit the antenna directivity to design a fast-converging ST processor for DS-CDMA.

A post-despread RAKE receiver is employed in this paper. We assume that the signal of interest (SOI) is the first user. The arriving signals are despread by passing them through a filter matched to the first user's spreading code. Next, the post-despread output is sampled in time to obtain different fingers of the RAKE receiver. The sampled output is then

$$
\mathbf{y}(q)=\mathbf{y}_{s}(q)+\mathbf{y}_{I}(q)+\mathbf{y}_{n}(q)
$$


where $\mathbf{y}_{s}(q)$ is the sampled output due to the desired user, $\mathbf{y}_{I}(q)$ is the sampled output due to interfering users, and $\mathbf{y}_{n}(q)$ is the sampled output due to white receiver noise. Note that the samples $\mathbf{y}_{n}(q)$ are temporally correlated due to the despreading operation. Let $Q$ be the number of temporal fingers collected by the receiver. There will be $M Q$ samples in a single ST snapshot. For the purpose of further processing, the $M Q$ samples are formed into an $M Q$-dimensional ST snapshot data vector

$$
\overline{\mathbf{y}}=\overline{\mathbf{y}}_{s}+\overline{\mathbf{y}}_{I}+\overline{\mathbf{y}}_{n}
$$

by augmenting the columns of the $M \times Q$ data into an $M Q \times 1$ vector. The covariance matrix $\mathbf{R}_{N}$ is

$$
\mathbf{R}_{N}=\mathbf{E}\left[\overline{\mathbf{y}}_{n} \overline{\mathbf{y}}_{n}^{H}\right]=\mathbf{I}_{M} \otimes \mathbf{R}_{1}
$$

where

$$
\mathbf{R}_{1}=\text { toeplitz }\left[R_{1,1}(0), \cdots, R_{1,1}(Q-1)\right]
$$

$\mathbf{I}_{M}$ is the $M \times M$ identity matrix, $\otimes$ means Kronecker product, $(\cdot)^{H}$ denotes the conjugate transpose operation, and $\mathbf{R}_{1,1}(q)$ is the sampled autocorrelation function of the first user's spreading code.

We use the output SINR as our performance metric because the metrics of SINR and symbol detection are equivalent in Gaussian interference, which is approximately the case when certain conditions are met, including the number of interfering users being large. These conditions are explained in [21]. The optimum output (in terms of the average SINR) is obtained by [22]

$$
y_{o}=\mathbf{w}^{H} \overline{\mathbf{y}}
$$

where $\mathbf{w}$ is the optimum ST beamformer, which is computed as $\mathbf{w}=\mathbf{R}_{\mathrm{IN}}^{-1} \overline{\mathbf{y}}_{s} . \mathbf{R}_{\mathrm{IN}}$ is the interference covariance matrix that consists of contributions from both white noise and undesired users. For independent user signals and noise

$$
\mathbf{R}_{\mathrm{IN}}=\mathbf{E}\left[\left(\overline{\mathbf{y}}_{I}+\overline{\mathbf{y}}_{n}\right)\left(\overline{\mathbf{y}}_{I}+\overline{\mathbf{y}}_{n}\right)^{H}\right]=\mathbf{R}_{I}+\mathbf{R}_{N}
$$

where $\mathbf{R}_{I}$ is the covariance matrix of the data due to interfering users after the despreading operation. Knowledge of $\mathbf{R}_{\mathrm{IN}}$ is required to perform optimum receiving. However, $\mathbf{R}_{\mathrm{IN}}$ is usually not available and must be estimated. Additional issues concerning this estimation will be discussed in Section IV.

\section{IDEAL PERFormanCE COMPARISON OF LINEAR AND CIRCULAR ARRAYS}

In this section, we compare linear and circular arrays and omnidirectional and directional antennas in a rich-scattering indoor environment. We assume for now that the interference covariance matrix is known so that optimum receiving can be performed. The baseline singleantenna power pattern $P_{b}(\theta)$ is adapted from the dual exponentially tapered slot antenna of [23]. To obtain antenna patterns of different directivities $D$, the baseline power pattern is modified as $|f(\theta)|^{2}=$ $P_{L}(\theta)=\left[P_{b}(\theta) \cos (\theta)\right]^{\xi}$ for a linear array and as $|f(\theta)|^{2}=P_{C}(\theta)=$ $\left[P_{b}\left(\theta-\theta_{0}\right) \cos \left(\theta-\theta_{0}\right) \cos \left(\left(\theta-\theta_{0}\right) / 2\right)\right]^{\xi}$ for a circular array. For the circular array, $\theta_{0}$ defines the pointing angle of the element. The antenna pattern $P_{L}(\theta)$ is two sided so that the linear array will not be blind to a full hemisphere of arriving signals. The antenna pattern $P_{C}(\theta)$ for the circular array is one sided with backlobes, but the configuration of the circular array enables it to detect signals from all directions.

The exponent $\xi$ is chosen to achieve the desired antenna directivity, and the power patterns are normalized to ensure the same effective
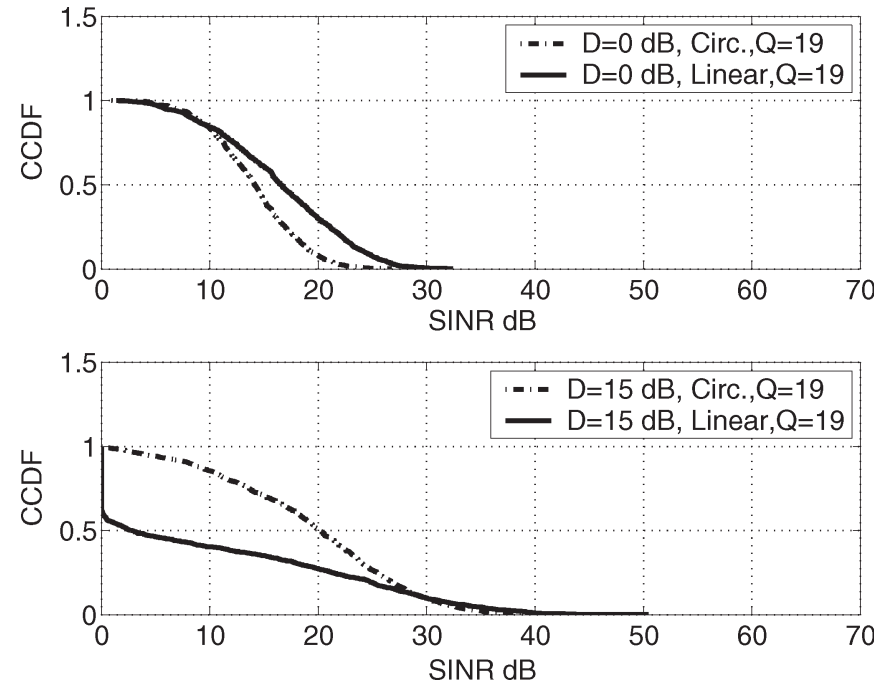

Fig. 1. Comparison of the SINR CCDF (probability of SINR > abscissa) for the linear and circular arrays at two different values of directivity. The number of simultaneous channel users is 19 .

isotropic radiated power for all directivities. The length of the linear array and the diameter of the circular array are both 3.5 wavelengths, and each array has eight antennas. For the linear array, the eight antennas are uniformly spaced and pointed to array boresight. The eight antennas of the circular array are uniformly spaced on a circle, and their maximum radiation is focused outward from the array center.

To demonstrate our approach, we simulated the modified Saleh-Valenzuela (SV) multipath propagation model [24]. According to the $\mathrm{SV}$ model, indoor multipath components arrive at the receiver in clusters, and every cluster consists of many rays. The time of arrival is described by two Poisson distributions. One distribution models the arrival time of clusters, and the other models the arrival time of rays within clusters. The AOA is independent of arrival time, and the AOA of all rays in one cluster can be modeled by a Laplacian distribution with standard deviation $\sigma$, and the mean AOA for a cluster is a random variable uniformly distributed on $[0,2 \pi)$. The parameters used in our simulations are $\Gamma=10 \mathrm{~ns}, \gamma=2 \mathrm{~ns}, 1 / \Lambda=25 \mathrm{~ns}, 1 / \lambda=2 \mathrm{~ns}$, and $\sigma=26^{\circ}$, where $\Gamma$ and $\gamma$ are the decay factors for clusters and rays in the same cluster, respectively, and $\Lambda$ and $\lambda$ are the cluster and ray arrival rates, respectively.

All signals are antipodal and spread with Gold codes of length 127 [1]. The power of the SOI is set to 1 , and the powers of interfering users are Rayleigh distributed with a mean of 1; therefore, the nearfar condition can be severe when the received power of interfering users are much higher than that of the desired user. The input signalto-noise-ratio (SNR) is SNR $=10 \log _{10}\left(P_{1} / N_{0}\right)=15 \mathrm{~dB}$. We configured the RAKE receiver to capture 20 temporal fingers. Under the aforementioned conditions, simulations were performed for linear and circular arrays with two different directivities: 1) $D=0 \mathrm{~dB}$ and 2) $D=15 \mathrm{~dB}$. The number of users $K$ was 19 , and the number of channel realizations was 1000 . In Fig. 1, we compare the complementary cumulative distribution functions (CCDFs) of the output SINR for omnidirectional and highly directive antennas. For example, Fig. 1 shows that when directive antennas are used, the linear array exceeds 10-dB SINR only about $40 \%$ of the time, whereas the circular array exceeds 10 -dB SINR over $80 \%$ of the time. Fig. 1 shows that for omnidirectional antennas, the linear array slightly outperforms the circular array. The linear array, however, is most affected by directivity: at high directivity, it has a higher probability of yielding a low SINR that can result in communication outage. 


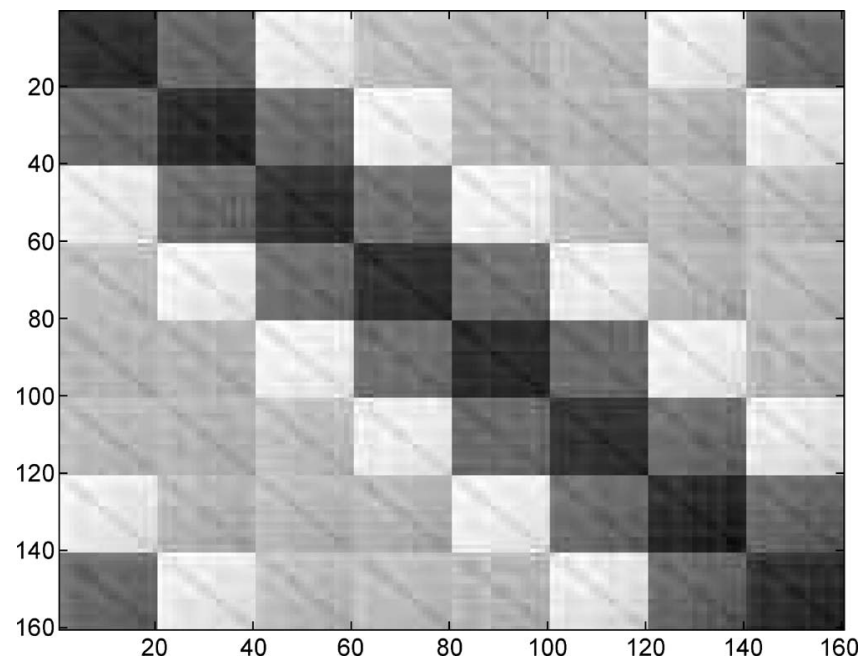

Fig. 2. Magnitude of the interference covariance matrix for a circular array with an element directivity of $10 \mathrm{~dB}$ (dark color indicates strong correlation). Note that the correlation is primarily localized to adjacent antennas.

These results are consistent with the different array structures. In the circular array, all eight antennas point to different directions spanning $\left[0^{\circ}, 360^{\circ}\right)$; therefore, for any AOA, the SOI can be collected by one or more antennas. In the linear array with directive antennas, the eight antennas point in the same direction. When the SOI arrives from that direction, all eight antennas receive it with large gain; hence, a large output SINR can be obtained. However, there are also cases where the SOI arrives from the endfire direction and all eight antennas receive it with low gain, which leads to many instances of low SINR.

\section{REDUCED-DIMENSION RECEIVER}

As aforementioned, $\mathbf{R}_{\mathrm{IN}}$ is usually unknown and must be estimated from received data, which results in an adaptive receiver. We propose a reduced-dimension receiving structure that improves adaptive convergence with little or no performance loss. To begin, we look at the structure of the interference covariance matrix $\mathbf{R}_{I}$ by considering the magnitude of the $\mathbf{R}_{I}$ entries under omnidirectional and directional assumptions. Fig. 2 shows the magnitude of the interference covariance matrix for an eight-element circular array with directive antennas, each collecting 20 temporal fingers. Thus, the dimension of the covariance matrix is $160 \times 160$. The covariance matrix can be treated as an $8 \times 8$ block matrix, where each block is of size $20 \times 20$. Block $(i, j)$ is the correlation of temporal fingers between the $i$ th and $j$ th antennas. In the omnidirectional case (not shown), all eight antennas are correlated, but in the directional case, only two adjacent antennas are closely correlated (one on each side). It is clear that directive antenna elements pointing in different directions produce a more localized spatial correlation structure than arrays that use omnidirectional antennas or antennas pointing in the same direction. Based on this observation, we now propose a two-stage receiver architecture that groups correlated antenna elements into a set of overlapping subarrays. For simplicity, we continue to consider a circular array, which ensures that the measurements collected on any antenna are most correlated with measurements collected on that antenna's nearest neighbors (as in Fig. 2). Assume that for a given directivity, measurements are strongly correlated over a span of $P$ adjacent antennas, which can be determined from knowledge of the antenna patterns and array configuration. We group the first $P(P<$ $M)$ adjacent antennas into the first subarray, then shift around the circular array to obtain the next subarray of $P$ antennas, and so on.

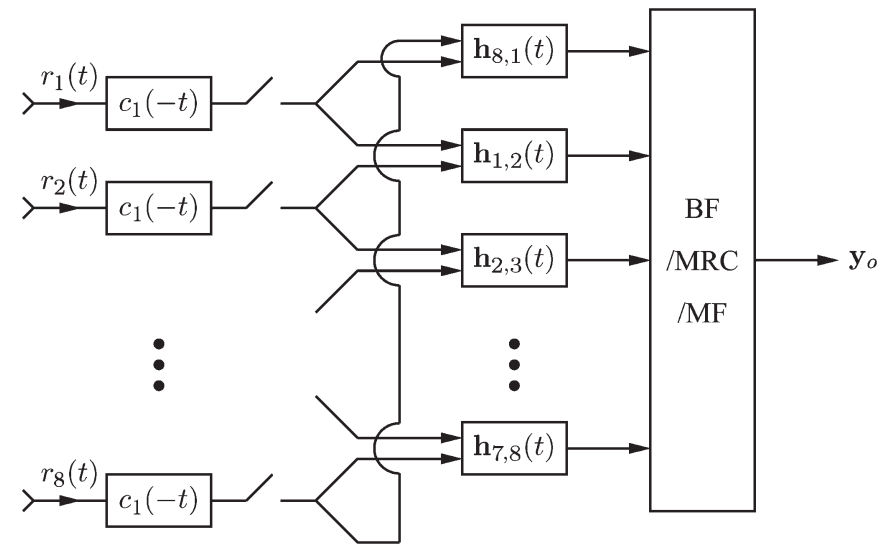

Fig. 3. Block diagram of the proposed reduced-dimension receiver with $L=$ 8 subarrays and $P=2$ elements per subarray.

A diagram of this process is shown in Fig. 3 for $P=2$ and $M=8$. For the results presented in this paper, the shift between subarrays is always one antenna, but in general, the shift can be larger. Each antenna in the array collects $Q$ temporal fingers; thus, each subarray forms a $P Q$-dimensional ST receiver. An FIR adaptive filter is applied to the $P Q$-dimensional data vector of each subarray to produce an output.

Let the fingers collected by the $p$ th antenna of the $i$ th subarray be the $Q \times 1$ vector $\mathbf{y}_{i, p}$, then the data vector for the $i$ th subarray is

$$
\overline{\mathbf{y}}_{i}=\operatorname{vec}\left(\left[\begin{array}{llll}
\mathbf{y}_{i, 1} & \mathbf{y}_{i, 2} & \cdots & \mathbf{y}_{i, P}
\end{array}\right]\right)
$$

where operator vec(.) transforms a $Q \times P$ matrix to a $Q P \times 1$ vector by appending its columns in order. Note that the data collected by an individual antenna will appear in multiple subarrays, but will be associated with a different value of $p$ due to its different location in each subarray. In addition, defining $\mathbf{w}_{i, p}$ to be the $Q \times 1$ vector of filter weights applied to the $p$ th antenna of the $i$ th subarray, the $i$ th subarray's FIR filter is

$$
\mathbf{w}_{i}=\operatorname{vec}\left(\left[\begin{array}{llll}
\mathbf{w}_{i, 1} & \mathbf{w}_{i, 2} & \cdots & \mathbf{w}_{i, P}
\end{array}\right]\right)
$$

and the output of the $i$ th subarray is $z_{i}=\mathbf{w}_{i}^{H} \overline{\mathbf{y}}_{i}$. The subarray filter of (10) is computed in the same way as the full-dimension filter of Section II; however, since $P<M$, fewer training data are required. Defining the matrix

$$
\mathbf{W}=\left[\begin{array}{cccccc}
\mathbf{w}_{1,1} & 0 & 0 & \ddots & \cdots & \mathbf{w}_{M, 2} \\
\vdots & \mathbf{w}_{2,1} & 0 & \cdots & \mathbf{w}_{M-1, P} & \vdots \\
\mathbf{w}_{1, P} & \vdots & \mathbf{w}_{3,1} & \cdots & 0 & \mathbf{w}_{M, p} \\
0 & \mathbf{w}_{2, P} & \vdots & \ddots & 0 & 0 \\
0 & 0 & 0 & \cdots & \mathbf{w}_{M-1,2} & \mathbf{w}_{M, 1}
\end{array}\right]
$$

the vector of the first-stage outputs is $\mathbf{z}=\mathbf{W}^{H} \overline{\mathbf{y}}$, and the interference covariance matrix of the first-stage outputs is

$$
\mathbf{R}_{\mathrm{IN}, z}=\mathbf{W}^{H} \mathbf{R}_{\mathrm{IN}} \mathbf{W}
$$

The elements of $\mathbf{z}$ are combined in the second processing stage to form a final scalar test statistic for bit detection. In the time-varying 

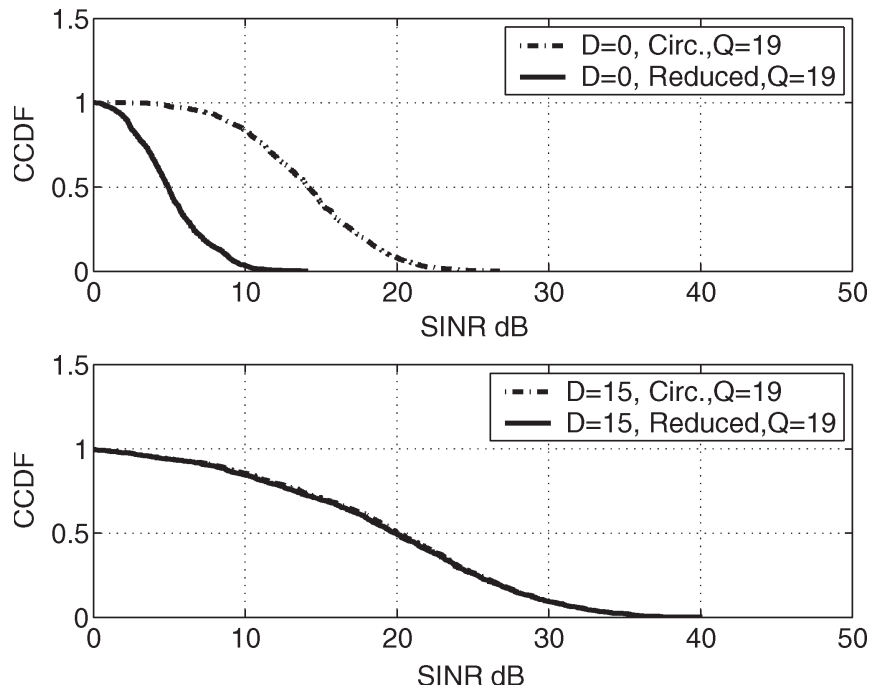

Fig. 4. SINR CCDF comparison for the circular array with full-dimension versus reduced-dimension architectures at two different values of directivity. At high directivity, the full- and reduced-dimension distributions are nearly identical.

channel simulations to follow, we compare three different combining strategies for the second stage. The first method is to perform BF [22] in the second stage using the propagated covariance structure of (12). The second method assumes that the first stage removes most of the interference such that maximal ratio combining (MRC) can be applied [12]. In this method, the second-stage filter is the principal generalized eigenvector of $\left[\mathbf{R}_{z}, \mathbf{R}_{\mathrm{IN}, z}\right]$, where $\mathbf{R}_{z}=\mathbf{E}\left[\mathbf{z z}{ }^{H}\right]$. The third method assumes that interference is completely removed in the first processing stage such that a matched filter (MF) can be applied. The signal impinging on each antenna is matched filtered with the spreading code of the desired user. The outputs of this bank of MFs are then passed to the first processing stage. The outputs of antennas 1 and 2 are passed to filter $\mathbf{h}_{12}$. The next subarray comprises antennas 2 and 3 , whose data are passed to filter $\mathbf{h}_{23}$, and so on until obtaining the final subarray comprising antennas 8 and 1 . The outputs of the eight subarrays are subsequently combined in the second processing stage using either the BF, MRC, or MF technique.

\section{A. Time-Invariant Channel}

In a time-invariant channel, channel statistics do not change with time; thus, covariance estimates can be very accurate. To isolate the effects of array configuration and antenna directivity, we first assume that the ideal $\mathbf{R}_{\mathrm{IN}}$ is available. Fig. 4 compares simulation results for the reduced- and full-dimension receivers. The results demonstrate that with omnidirectional antennas, the full-dimension receiver outperforms the reduced-dimension receiver. The reason is that for omnidirectional antennas, the interference covariance matrix structure does not match the architecture of the reduced-dimension receiver. However, as antenna elements become more directive, the correlation structure becomes similar to that shown in Fig. 2; thus, due to the proper match between the covariance and receiver structures, the performance of the reduced-dimension receiver approaches the performance of the ideal full-dimension receiver. In Fig. 4, when $D=15 \mathrm{~dB}$, the output SINR distribution of the reduced-dimension receiver is nearly identical to that of the full-dimension receiver. The benefit of the matched reduced-dimension architecture, however, is improved convergence of the adaptive processor, which is demonstrated next.

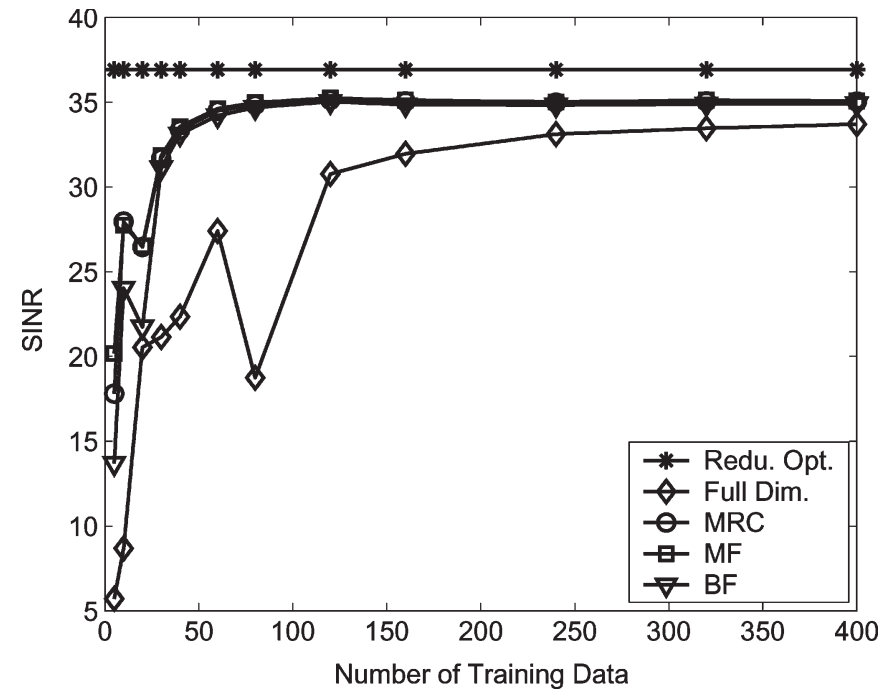

Fig. 5. Average output SINR versus amount of training data in a time-varying environment. Comparisons are made between optimum filtering, adaptive fulldimension filtering, and adaptive reduced-dimension filtering with various second-stage combining approaches.

\section{B. Time-Varying Channel}

In time-varying channels, because of the movement of the transmitter, receiver, and reflectors, channel statistics change with time. Therefore, it is necessary to continuously train the adaptive receiver to keep up with the time-varying covariance matrix. The main issue that arises when trying to estimate the interference covariance matrix is that the matrix must be trained from data that are essentially free of energy from the desired user. If coarse symbol synchronization is available, these signal-free data can be obtained by collecting temporal samples after the last multipath delay is expected to arrive in each bit interval. If no multipath components due to the desired user are arriving in a given time window, then the output of the despreading operation will be free of signal energy, except for some small amount due to nonzero autocorrelation of the spreading code at large time lags. These interference-only data can be used to estimate ST interference and noise statistics, but the amount of training data available in a single bit interval is likely insufficient. Furthermore, if the channel is time varying, then the number of previous bit intervals with statistically identical data depends on the rate of change of the channel. In this paper, we assume that training must be performed over multiple bit intervals according to

$$
\tilde{\mathbf{R}}_{\mathrm{IN}, k}=\frac{1}{N} \sum_{j=1}^{N} \mathbf{y}_{\mathrm{IN}, k-j} \mathbf{y}_{\mathrm{IN}, k-j}^{H}
$$

where $N$ is the number of training data vectors, $\tilde{\mathbf{R}}_{\mathrm{IN}, k}$ is the estimated interference and noise covariance for the current ( $k$ th) bit, and $\mathbf{y}_{\mathrm{IN}, k-j}$ is the interference-and-noise-only data vector collected at $j$ bit intervals prior to the current bit. A similar time-averaging approach has been taken in [25].

It is well known that for an $M$-dimensional covariance matrix, a good estimate can be obtained when the number of statistically independent and identically distributed training data snapshots is $N \geq$ $2 M[19]$. Since the required training data are proportional to the size of the covariance matrix, the full-dimension ST receiver needs much more training data and a much longer training process. When the channel is time varying, the channel statistics may change during the training process, and the estimation will be degraded, which will result in degraded performance. 


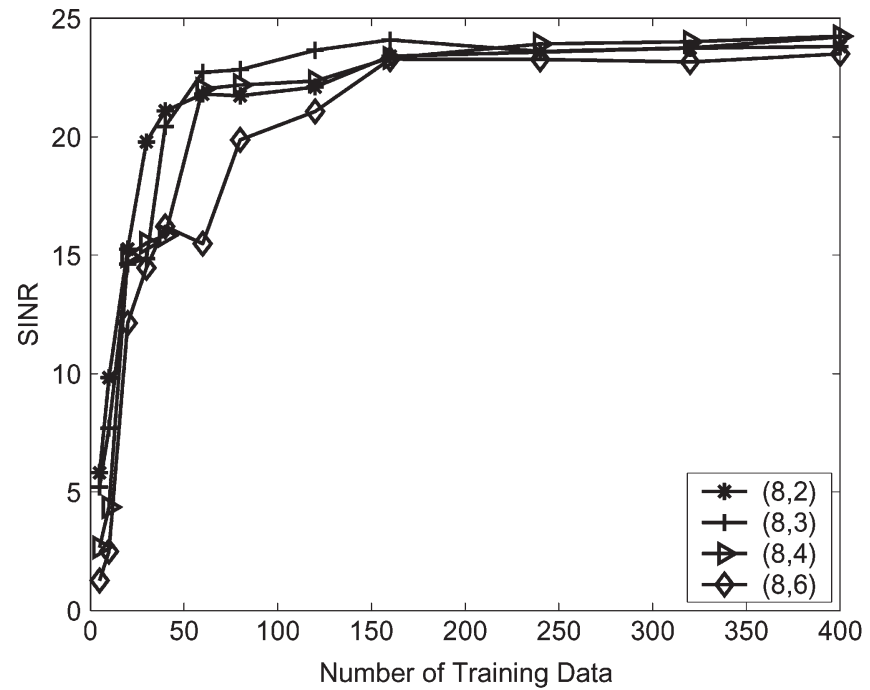

(a) $\mathrm{D}=3 \mathrm{~dB}$

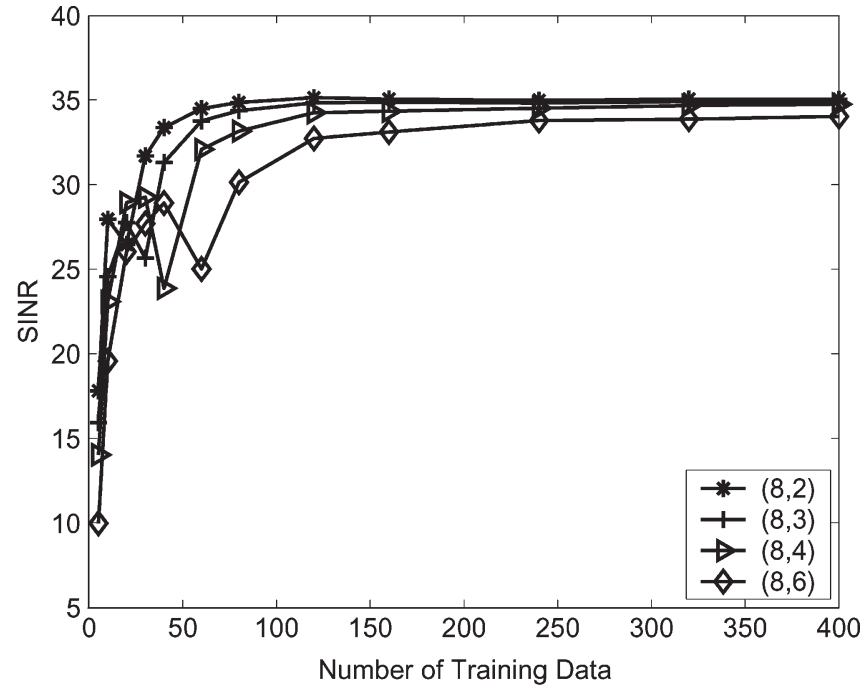

(b) $\mathrm{D}=15 \mathrm{~dB}$

Fig. 6. For the reduced-dimension architecture, a comparison of the average output SINR versus training data parameterized by subarray structure. (a) At low directivity, the best-performing structure is unclear. (b) At high directivity, the architecture with matched subarray size $(P=2)$ performs best.

We use the time-varying channel of [26] to compare the performance of the full- and reduced-dimension receivers in a timevarying environment. In this channel model, multipath components only exist for a finite period of time, and their delay, decay, and AOA are changing with time. The appearance and disappearance of the multipath components are modeled by a birth-death process: the multipath components appear with birth rate $\lambda_{B}$ and disappear with death rate $\lambda_{D}$. Parameters $\lambda_{B}$ and $\lambda_{D}$ are related to the environment. In this paper, we select one pair of values from [26]: $\lambda_{B}=9.39 / \mathrm{m}$, and $\lambda_{D}=0.53 / \mathrm{m}$. The parameters modeling the change of delay, decay, and AOA are set as $\tau_{A}=5.79 \mathrm{~ns}, \tau_{B}=27.86 \mathrm{~ns}, q_{\tau}=0.045$, $\tau_{\text {Max }}^{\prime}=297.28 \mathrm{~ns}, \vartheta_{a}=12.01^{\circ}$, and $\tau_{\vartheta}=6.87 \mathrm{~ns}$, where $\tau_{A}$ and $\tau_{B}$ are time constants for power decay, $q_{\tau}$ is the quotient of the exponential decay functions, $\tau_{\text {Max }}^{\prime}$ is the maximum multipath delay, and $\vartheta_{a}$ and $\tau_{\vartheta}$ are parameters to model the AOA. In this configuration, the number of the multipath components will change on average about every 130 bits. In our simulations, the receiver captures ten temporal fingers, and the results are averaged over 200 Monte Carlo runs. For the purpose of comparison, optimum reduced-dimension $\mathrm{BF}$ (no training) is performed based on the ideal interference and noise statistics. Optimum reduced-dimension results are labeled as "Redu. Opt."

Fig. 5 shows the performance of the full- and reduced-dimension receivers versus training data support. The interference-plus-noise covariance matrix is $80 \times 80$ for the full-dimension receiver and $20 \times 20$ for the reduced-dimension receiver. We see that when the training number is low (around 50), the reduced-dimension receiver achieves a much higher output SINR than the full-dimension receiver. The reduced-dimension receiver has a significant advantage in terms of covariance estimation. In fact, even when the training number is high (400), the full-dimension receiver still cannot perform as well as the reduced-dimension receiver. This is because by the time the full-dimension receiver obtains enough training data, the channel has already changed. In Fig. 5, it can also be noted that the three curves for the reduced-dimension receiver eventually converge when the training data number is large. This implies that a well-trained first stage is successful at removing most of the MAI.

In Fig. 5, we configured the reduced-dimension receiver as in Fig. 3, but this may not always be optimum. To explore the effects of different reduced-dimension receiver configurations, we define a receiver structure according to the pair $(L, P)$, where $L$ is the number of subarrays, and $P$ is the number of antennas in each subarray. For example, the reduced-dimension receiver shown in Fig. 3 is represented by $(8,2)$. It should be noted that a small $P$ implies an advantage in covariance estimation, but interference rejection capability may be diminished if $P$ is chosen too small for the covariance structure.

Since the covariance matrix structure is related to the antenna directivity, one reduced-dimension configuration cannot match the covariance matrix structure for all antenna directivities. To show this, in Fig. 6, we show results for reduced-dimension architectures of different configurations for $D=3 \mathrm{~dB}$ and $D=15 \mathrm{~dB}$. In Fig. 6, we show performance versus training data for ST BF in the first stage and MRC combining in the second stage. For $D=3 \mathrm{~dB}$, the $(8,2)$ configuration performs best for less than 50 training data snapshots because it requires the least amount of training data. However, the $(8,2)$ configuration is not well matched to the correlation structure; thus, as the number of training snapshots exceeds 50, configurations with more antennas per subarray perform better. Specifically, the $(8,3)$ configuration performs best if the number of snapshots is between 50 and 200 , and the $(8,4)$ configuration performs best for over 200 training snapshots. Although the $(8,6)$ configuration is best matched to the spatial correlation structure, this configuration requires too much training data for a time-varying environment. By the time the $(8,6)$ configuration collects enough training data to properly estimate the interference and noise statistics, the multipath environment has changed.

For $D=15 \mathrm{~dB}$, the $(8,2)$ configuration again performs best for small amounts of training data. For directive antennas, the $(8,2)$ configuration is also well matched to the spatial correlation structure of the data. Hence, the $(8,2)$ configuration remains the best performer even for larger amounts of training data. We also note that compared to each other, the performance of the system with directive antennas is better than the system with nearly omnidirectional antennas. For this propagation environment, directive antennas boost performance through improved convergence, which is achieved through proper matching of processing architecture to a localized correlation structure. 


\section{CONCLUSiON}

With directive antennas in a circular or conformal arrangement, the interference covariance matrix is more "locally" correlated than for an array of omnidirectional antennas. This localized correlation structure allows the receiving array to be divided into several reduced-dimension subarrays in parallel. Simulation results have shown that in a timevarying environment, the reduced-dimension architecture outperforms the full-dimension architecture due to the reduced amount of training data required for training the adaptive processor.

\section{REFERENCES}

[1] A. J. Viterbi, CDMA: Principles of Spread Spectrum Communication. Reading, MA: Addison-Wesley, 1999.

[2] J. Barry, E. Lee, and D. Messerschmitt, Digital Communication, 3rd ed. Boston, MA: Kluwer, 2004.

[3] R. Price and P. Green, "A communication technique for multipath channels," Proc. IRE, vol. 46, no. 3, pp. 555-570, Mar. 1958.

[4] A. Sayeed, A. Sendonaris, and B. Aazhang, "Multiuser detection in fastfading multipath environments," IEEE J. Sel. Areas Commun., vol. 16, no. 9, pp. 1691-1701, Dec. 1998.

[5] Z. Zvonar and D. Brady, "Linear multipath-decorrelating receivers for CDMA frequency-selective fading channels," IEEE Trans. Commun., vol. 44, no. 6, pp. 650-653, Jun. 1996.

[6] B. Suard, A. Naguib, G. Xu, and A. Paulraj, "Performance of CDMA mobile communication systems using antenna arrays," in Proc. ICASSP, Apr. 1993, pp. 153-156.

[7] A. Naguib, A. Paulraj, and T. Kailath, "Capacity improvement with basestation antenna arrays in cellular CDMA," IEEE Trans. Veh. Technol., vol. 43, no. 3, pp. 691-698, Aug. 1994.

[8] R. Kohno, H. Imai, and S. Pasupathy, "Combination of an adaptive array antenna and a canceller of interference for direct-sequence spreadspectrum multiple-access system," IEEE J. Sel. Areas Commun., vol. 8, no. 4, pp. 675-682, May 1990.

[9] J. Ramos, M. Zoltowski, and H. Liu, "Low-complexity space-time processor for DS-CDMA communications," IEEE Trans. Signal Process., vol. 48 , no. 1, pp. 39-52, Jan. 2000

[10] T. Lee and T. Tsai, "A beamspace-time interference canceling CDMA receiver for sectored communications in a multipath environment," IEEE J. Sel. Areas Commun., vol. 19, no. 7, pp. 1374-1384, Jul. 2001.

[11] E. Onggosanusi, A. Sayeed, and B. Van Veen, "Canonical space-time processing for wireless communications," IEEE Trans. Commun., vol. 48, no. 10 , pp. $1669-1680$, Oct. 2000

[12] T. Wong, T. Lok, J. Lehnert, and M. Zoltowski, "A linear receiver for direct-sequence spread-spectrum multiple-access systems with antenna arrays and blind adaptation," IEEE Trans. Inf. Theory, vol. 44, no. 2, pp. 659-676, Mar. 1998.

[13] A. Klein, G. Kaleh, and P. Baier, "Zero forcing and minimum mean-square-error equalization for multiuser detection in code-division multiple-access channels," IEEE Trans. Veh. Technol., vol. 45, no. 2, pp. 276-287, May 1996.

[14] H. Liu and M. Zoltowski, "Blind equalization in antenna array CDMA systems," IEEE Trans. Signal Process., vol. 45, no. 1, pp. 161-172, Jan. 1997.

[15] I. Ghauri and D. Slock, "Linear receivers for the DS-CDMA downlink exploiting orthogonality of spreading sequences," in Proc. Conf. Rec. 32nd Asilomar Conf. Signals, Syst., Comput., Nov. 1998, vol. 1, pp. 650-654.

[16] A. M. Haimovich, A. Shah, and X. Wu, "Reduced-rank array processing for wireless communications with applications to IS-54/IS-136," IEEE Trans. Commun., vol. 48, no. 5, pp. 743-747, May 2000.

[17] J. Goldstein and I. Reed, "Reduced-rank adaptive filtering," IEEE Trans. Signal Process., vol. 45, no. 2, pp. 492-496, Feb. 1997.

[18] C. Dietrich, K. Dietze, J. Nealy, and W. Stutzman, "Spatial, polarization, and pattern diversity for wireless handheld terminals," IEEE Trans. Antennas Propag., vol. 49, no. 9, pp. 1271-1281, Sep. 2001.

[19] I. Reed, J. Mallett, and L. Brennan, "Rapid convergence rate in adaptive arrays," IEEE Trans. Aerosp. Electron. Syst., vol. AES-10, no. 6, pp. 853863, Nov. 1974.

[20] W. Stutzman and G. Thiele, Antenna Theory and Design, 2nd ed. New York: Wiley, 1998.
[21] L. R. Pickholtz, L. Milstein, and D. Schilling, "Spread spectrum for mobile communications," IEEE Trans. Veh. Technol., vol. 40, no. 2, pp. 313 322, May 1991

[22] S. Haykin, Adaptive Filter Theory, 4th ed. Upper Saddle River, NJ: Prentice-Hall, 2001.

[23] M. Greenberg, K. Virga, and C. Hammond, "Performance characteristics of the dual exponentially tapered slot antenna (DETSA) for wireless communications applications," IEEE Trans. Veh. Technol., vol. 52, no. 2, pp. 305-312, Mar. 2003.

[24] Q. Spencer, B. Jeffs, M. Jensen, and A. Swindlehurst, "Modeling the statistical time and angle of arrival characteristics of an indoor multipath channel," IEEE J. Sel. Areas Commun., vol. 18, no. 3, pp. 347-360, Mar. 2000.

[25] J. Ramos and M. Zoltowski, "Reduced complexity blind 2D RAKE receiver for CDMA," in Proc. 8th IEEE Signal Process. Workshop Statistical Signal Array, Jun. 1996, pp. 502-505.

[26] T. Zwick, C. Fischer, and W. Wiesbeck, "A stochastic multipath channel model including path directions for indoor environments," IEEE J. Sel. Areas Commun., vol. 20, no. 6, pp. 1178-1192, Aug. 2002.

\section{Wireless Data Traffic Estimation Using a State-Space Model}

Farzaneh Kohandani, Member, IEEE, Derek W. McAvoy, and Amir K. Khandani, Member, IEEE

Abstract-A new forecasting technique called the extended structural model (ESM) is presented. This technique is derived from the basic structural model (BSM) by the introduction of extra parameters that were assumed to be 1 in the BSM. The ESM is constructed from the training sequence using standard Kalman filter recursions, and then, the extra parameters are estimated to minimize the mean absolute percentage error (MAPE) of the validation sequence. The model is evaluated by the prediction of the total number of minutes of wireless airtime per month on the Bell Canada network. The ESM shows an improvement in the MAPE of the test sequence over the BSM, seasonal autoregressive integrated moving average (ARIMA), and generalized random walk models on the series considered in this paper. The improved prediction can significantly reduce the cost for wireless service providers who need to accurately predict future wireless spectrum requirements.

Index Terms-Autoregressive integrated moving average (ARIMA), basic structural model (BSM), Kalman filter, mean absolute percentage error (MAPE).

\section{INTRODUCTION}

Accurate forecasting is crucial for many organizations and plays an important role in most decision-making processes. To generate predictions, information about past events, which is called "time series data," is collected. There are four general pattern types for time series data: stationary, trend, seasonal, and cyclical [1].

Autoregressive integrated moving average (ARIMA) methods are advanced models for prediction that integrate an autoregressive model and a moving average model [2], [3]. Yin et al. [4] have introduced a

Manuscript received August 9, 2005; revised October 10, 2006, March 16, 2007, May 28, 2007, and June 28, 2007. First published April 18, 2008; current version published November 12, 2008. The review of this paper was coordinated by Prof. C. Lin.

F. Kohandani and A. K. Khandani are with the Department of Electrical and Computer Engineering, University of Waterloo, Waterloo, ON N2L 3G1, Canada (e-mail: farzaneh@cst.uwaterloo.ca; khandani@cst.uwaterloo.ca).

D. W. McAvoy is with the Technology Planning Team, Bell Mobility, Mississauga, ON L4W 5J4, Canada (e-mail: derek.mcavoy@bell.ca).

Color versions of one or more of the figures in this paper are available online at http://ieeexplore.ieee.org.

Digital Object Identifier 10.1109/TVT.2008.923663 